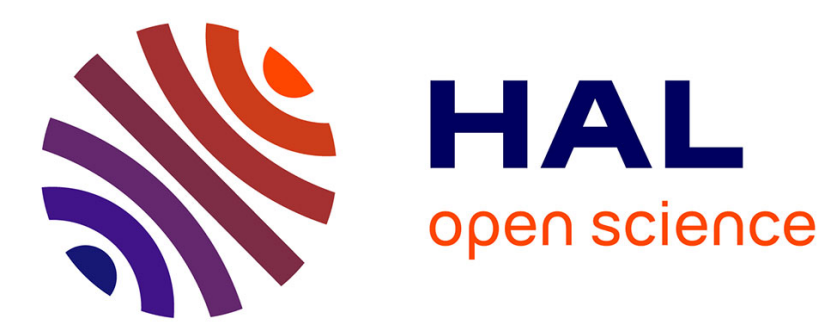

\title{
L'ALCOVE ET LA COUETTE
}

Béatrice Damian-Gaillard, Guillaume Soulez

\section{To cite this version:}

Béatrice Damian-Gaillard, Guillaume Soulez. L'ALCOVE ET LA COUETTE. Réseaux: communication, technologie, société, 2001, 10.3917/res.105.0101 . hal-02608277

\section{HAL Id: hal-02608277 \\ https://hal.science/hal-02608277}

Submitted on 16 Oct 2020

HAL is a multi-disciplinary open access archive for the deposit and dissemination of scientific research documents, whether they are published or not. The documents may come from teaching and research institutions in France or abroad, or from public or private research centers.
L'archive ouverte pluridisciplinaire HAL, est destinée au dépôt et à la diffusion de documents scientifiques de niveau recherche, publiés ou non, émanant des établissements d'enseignement et de recherche français ou étrangers, des laboratoires publics ou privés. 


\section{L'ALCOVE ET LA COUETTE}

Presse féminine et sexualité : l'expérience éphémère de bagatelle (1993-1994)

Béatrice Damian-Gaillard, Guillaume Soulez

\section{La Découverte | « Réseaux »}

2001/1 n 105 | pages 101 à 129

ISSN 0751-7971

Article disponible en ligne à l'adresse :

http://www.cairn.info/revue-reseaux-2001-1-page-101.htm

\section{!Pour citer cet article :}

Béatrice Damian-Gaillard, Guillaume Soulez, «L'alcove et la couette. Presse féminine et sexualité : l'expérience éphémère de bagatelle (1993-1994) », Réseaux 2001/1 (nº 105), p. 101-129.

DOI 10.3917/res.105.0101

Distribution électronique Cairn.info pour La Découverte.

(C) La Découverte. Tous droits réservés pour tous pays.

La reproduction ou représentation de cet article, notamment par photocopie, n'est autorisée que dans les limites des conditions générales d'utilisation du site ou, le cas échéant, des conditions générales de la licence souscrite par votre établissement. Toute autre reproduction ou représentation, en tout ou partie, sous quelque forme et de quelque manière que ce soit, est interdite sauf accord préalable et écrit de l'éditeur, en dehors des cas prévus par la législation en vigueur en France. Il est précisé que son stockage dans une base de données est également interdit. 


\section{L'ALCOVE ET LA COUETTE}

Presse féminine et sexualité : l'expérience éphémère de Bagatelle (1993-1994)

Béatrice DAMIAN-GAILLARD

Guillaume SOULEZ 


\begin{abstract}
«Etant donné la similarité entre les situations narrées et les situations réelles des lectrices qu'on peut reconstruire à partir d'études sur le public, ces récits mimétiques sont certainement capables d'offrir au moins une régulation imaginaire des identités ${ }^{1}$.»
\end{abstract}

$\mathrm{O}$ ctobre 2000, sur un quai de métro parisien, une série de panneaux insolite: un homme se déshabille, enlevant progressivement, d'affiche en affiche, ses sous-vêtements Lacoste. Comment en eston arrivé là ? La possibilité d'exposer au regard public un homme nu a peutêtre été préparée par l'évolution de la presse féminine sur la question de la sexualité, dont témoigne aujourd'hui les couvertures des magazines qui offrent souvent aux regards des corps masculins et féminins dénudés. Aux débuts des années 1990, distribué en kiosque, Bagatelle, qui fait figure de précurseur, proposa une formule plus osée encore en se lançant dans l'effeuillage de jeunes mannequins hommes en pages centrales. Ce striptease masculin constituait le clou d'un magazine consacré à la sexualité, mais qui s'inscrivait clairement dans le champ de la presse féminine grand public. Cette expérience, qui dura un an environ ${ }^{2}$, était semble-t-il inédite : en s'emparant, de façon en apparence provocante, de la question du désir et du plaisir féminins, elle interrogeait - et elle interroge encore - les limites et les tabous de la presse féminine. Comment un magazine a-t-il pu concilier les conventions de la presse féminine avec un tel projet? Le propos de cette étude est double: à travers l'expérience de Bagatelle, il s'agit de comprendre les difficultés de la presse féminine à traiter de la sexualité et de

1. RUNTE-HELBING, 1986.

2. Né en novembre 1993, ce journal publia quatre numéros jusqu'en juin 1994. Bagatelle a été édité par le groupe BCMC, une SARL parisienne dont le capital se montait à $100000 \mathrm{~F}$. Le premier numéro annonce une périodicité mensuelle, démentie par les trois numéros suivants, bimensuels (janvier/février, mars/avril et mai/juin 1994). 
s'interroger sur les discours que construit la presse féminine sur les rôles sociaux des femmes et des hommes à travers cette question de la sexualité.

L'appellation de «presse féminine » suscite des interrogations, voire des polémiques ${ }^{3}$. Elles portent sur les mécanismes de construction d'une identité à partir d'un référent sexuel, sur les représentations qui sont données des rapports entre (les) homme(s) et (les) femme(s), et sur les relations de cette presse avec les évolutions sociales (la place des femmes dans la société, la question des modalités de la médiatisation de la parole des femmes, etc.). Il s'agit précisément ici d'analyser un cas particulier, hors des catégories habituelles du secteur, pour mieux observer celui-ci. Prenant le parti du plaisir féminin en s'approchant, en apparence, du magazine de charme, Bagatelle est, en effet, un journal qui se joue des frontières de la presse féminine «classique ». Féminin et grivois, renvoyant aux jeux de l'amour considérés comme une activité légère et insouciante, son titre indique d'emblée une ambivalence : a-t-on affaire à une revue érotique destinée à un lectorat féminin, ou à un magazine féminin qui cherche à aborder sans fard la sexualité des femmes? Bagatelle fut présenté par sa rédactrice en chef, Denyse Beaulieu, à la fois comme la version magazine des spectacles de charme adressés aux femmes (comme les «Chippendales»), et comme un magazine féminin grand public, tel Cosmopolitan par exemple. De fait, si le numéro un se voulait «le magazine de la femme sensuelle », les suivants précisèrent le positionnement éditorial du journal dans la sphère de la presse magazine féminine en le proclamant «le magazine féminin qui déshabille les hommes ».

C'est cette tension entre une ligne éditoriale inédite en France et le cadre qu'impose son ancrage dans l'univers de la presse féminine qui constitue pour nous l'enjeu du projet de Bagatelle. Nous voulons voir comment

3. Ces interrogations tournent, pour la plupart, autour de la définition à donner au terme «féminin» et de la possibilité d'une «identité féminine» définie comme construction historique et sociale ou, au contraire, comme réalité biologique («nature »). Formulées par les féministes à partir des années 1970, elles ont pointé le rôle des médias, et plus particulièrement de la presse féminine dans la construction de stéréotypes entravant la prise en compte politique et sociale de la condition féminine. Un ouvrage emblématique de la façon dont ces questions étaient posées sur le mode polémique est celui d'Anne-Marie Dardigna (DARDIGNA, 1974). Pour notre part, nous limitons l'emploi de l'expression «presse féminine" au média écrit qui a pour caractéristique principale d'avoir un lectorat très majoritairement constitué de femmes (ce qui nourrit parfois chez les acteurs professionnels la croyance en l'existence d'une « communauté » féminine). 
Bagatelle traverse les frontières traditionnelles de la presse féminine, tout en témoignant des évolutions mêmes de ce secteur. De ce point de vue, nous faisons nôtre la démarche d'E. Véron, pour qui «les propriétés 'sémiologiques' des discours n'ont aucun intérêt en elles-mêmes, [elles ne] sont que les produits (plus ou moins stabilisés) de la sémiosis socioculturelle », ce qui permet dès lors de penser la construction discursive comme résolution de tensions nées de la concurrence entre supports, dans la mesure où il s'agit de «mouvements de convergence entre la production et la réception de discours [...] mouvements dynamisés par la recherche permanente d'articulation entre l'offre et la demande ${ }^{4}$ ». En tant qu'instance de discours, Bagatelle se trouve donc ainsi au centre d'un processus sémiotique complexe. Ce magazine doit proposer un discours, c'est-à-dire pour E. Véron une posture énonciative identifiée, autour de la sexualité féminine, dont la fonction est de susciter l'intérêt d'un lectorat, mais en respectant les contraintes culturelles et économiques de la sphère de production dans laquelle il s'inscrit, tout en s'appropriant les évolutions sociales en cours (dont témoigne par exemple le succès des spectacles de Chippendales), dans la mesure où ces évolutions interfèrent sans cesse sur les modalités de construction et d'interprétation des signes élaborés dans les discours médiatiques. Nous allons voir ainsi que deux mouvements contribuent à produire l'identité énonciative de Bagatelle, une logique de justification et une logique d'innovation: pour être identifié et pour être entendu, le magazine doit à la fois défendre, au sein même de ses colonnes, l'originalité de son projet, et proposer des formes inédites de discours sur la sexualité pour relever le défi qu'il se propose.

La volonté d'inscription au sein de la presse féminine suppose, en effet, à la fois de composer avec les représentations «classiques» de la femme présentes dans les autres titres féminins, et de s'en affranchir sans pour autant empêcher l'adhésion du lectorat ${ }^{5}$. Nous tenterons de mettre au jour ce qui permet à Bagatelle de revendiquer un «nouveau » discours féminin sur le sexe notamment à travers la promotion d'un certain «droit au plaisir». Sous cet angle, l'analyse de l'ethos des rédacteurs permet de préciser la

4. VERON, 1994 p, 62.

5. Des entretiens effectués en 1993/94, auprès des professionnels impliqués dans la conception de magazines féminins (Marie Claire, ELLE, Cosmopolitan, Modes et travaux et Femme actuelle), nous serviront de point de référence pour interpréter les stratégies de légitimation développées par Bagatelle. L'intégralité des entretiens est retranscrite dans DAMIAN, 1995 (annexes). 
posture énonciative adoptée dans ses articles par le magazine, puisque nous nous demanderons comment l'affichage par les journalistes d'une position «subjective» et professionnelle vis-à-vis de la sexualité contribue à légitimer l'existence du magazine ${ }^{6}$.

Cette recherche de légitimation se retrouve dans les tentatives de Bagatelle de faire exister un «nouveau » discours sur la sexualité des femmes. De par son projet, en effet, le magazine est voué à innover dans ce domaine, c'est-àdire à proposer de «nouveaux » rôles féminins, de nouvelles relations entre hommes et femmes, afin de manifester de façon tangible l'écart qu'il prétend creuser par rapport à la timidité habituelle de la presse féminine sur ces questions. Pour décrypter cette tentative de «reformulation» de ces représentations, nous nous appuierons sur une approche sociologique des scénarios cognitifs, ou «scripts », qui déterminent dans la vie courante les conduites sexuelles 7 . Nous observerons depuis l'analyse de ces récits comment Bagatelle peut parvenir à se singulariser en parlant de la sexualité des femmes, en particulier en proposant à celles-ci de nouvelles places dans la réalisation des scénarios du désir. Mais le journal rencontre rapidement et fortement les normes traditionnellement véhiculées par la presse féminine : nous verrons quelle forme prend alors le compromis échafaudé par Bagatelle, compromis révélateur de son positionnement et qui l'empêche peut-être de trouver son public.

En conclusion, nous nous intéresserons à la façon dont la presse féminine d'aujourd'hui traite de la question de la sexualité. Cette confrontation entre le projet de Bagatelle, expérience éphémère, et l'importance prise actuellement par les sujets sexuels dans les magazines féminins amène à s'interroger sur les raisons d'un échec. L'analyse approfondie des numéros de Bagatelle révèle, parallèlement à la construction d'un univers de discours spécifique, que la recherche incessante d'adéquation entre le support et son lectorat, non dissimulée par les stratégies de justification des responsables eux-mêmes, peut aussi s'analyser stricto sensu comme un révélateur de préoccupations liées à une démarche marketing (caractérisée par la mobilisation d'instruments de mesure comme les sondage, les études, etc.).

6. L'étude de l'ethos rhétorique pour analyser l'affichage public de positions socioprofessionnelles assumées au moment même de l'acte de discours a fait l'objet de propositions dans SOULEZ, 1998 et 2001.

7. GAGNON, 1999. 
Nous essaierons d'en formuler les visées stratégiques sous forme d'hypothèses ${ }^{8}$.

\section{LE «DROIT AU PLAISIR » : ENTRE POSTURE ENONCIATIVE ET POSTULATS DE LA PRESSE FEMININE}

Lancer un magazine consacré au plaisir féminin amène les responsables à justifier leur entreprise, dans la mesure où il apparaît nécessaire d'expliquer au lectorat féminin et aux annonceurs les raisons d'un projet atypique. Que signifie exactement la revendication de l'expression d'une sensualité féminine, et quel type d'objectif peut poursuivre un magazine féminin en se spécialisant ainsi ? Comment cette perspective rencontre-t-elle les caractéristiques traditionnelles de la presse féminine? Quelle posture adoptent alors les rédacteurs pour légitimer les choix de Bagatelle et susciter l'adhésion des lectrices?

Le discours de Bagatelle se caractérise à la fois par son inscription dans l'univers de la presse féminine, et sa forte démarcation sur la question du plaisir féminin. Ceci se voit, en premier lieu, dans le système de rubricage du magazine. Même s'il écarte les sujets liés à la famille, à la cuisine et à la décoration intérieure, Bagatelle reprend l'essentiel des rubriques traditionnelles de la presse féminine (mode, habillement, beauté, vie privée, séduction...), mais en transformant ces rubriques en lieux de traitement de problématiques liées, de près ou de loin, à la sexualité. Ainsi, le billet d'humeur, les rubriques consacrées au tourisme, à l'actualité des stars, à la mode, à la consommation en général, etc. sont toutes traversées par une perspective sexuelle ainsi que le courrier des lectrices, fait inédit dans la presse féminine généraliste. Les questions posées lors des interviews des personnalités féminines ou masculines portent principalement sur les aspects les plus intimes de leur vie. Même l'horoscope propose deux horizons de conseils adressés à la lectrice: l'un sentimental («côté cœur»), l'autre explicitement sexuel (« côté couette »).

Mais lorsque Bagatelle revendique un «droit au plaisir » féminin, il fait mine de reprendre à son compte le combat féministe des années 1970, lors

8. Cette approche des stratégies marketing comme outils et comme cadre conceptuel structurant les pratiques et les représentations des acteurs de la presse féminine a été proposée dans DAMIAN, 1995. 
de l'émergence des plannings familiaux et des mouvements de libération des femmes. En ce sens, ce droit peut apparaître comme la suite logique de la revendication des femmes «à disposer librement de leur corps». Les revendications féministes (qui trouvèrent à l'époque un écho dans la presse féminine généraliste) s'accompagnaient, toutefois, d'une mise en accusation des images et des discours construits sur la femme, ses rôles et sa place à la fois dans le couple, la famille et la société ${ }^{9}$, ce qui n'est pas très présent, ou très peu présent, dans Bagatelle. Ce discours «militant» de libération des femmes (via celle des hommes) se limite en fait à des préoccupations purement sensuelles. On le voit, de façon emblématique dans l'éditorial du premier numéro («Libérons-les, déshabillons-les!») dont l'allure militante est avant tout humoristique. Bagatelle défend le droit de montrer des hommes nus parce que d'autres l'ont fait (spectacles de strip-tease, cinéastes, publicitaires et presse anglophone), et tandis que certains continuent à affirmer que cela n'intéresse pas les femmes. Pour la rédactrice en chef, les rôles sociaux impartis aux femmes ont évolué, et leur capacité de «tout concilier» (travail, vie amoureuse et maternité) légitiment ce choix. Mais cet argument vise surtout à modifier les modes de séduction et les rapports sexuels entre les hommes et les femmes. «Libérer » les hommes se résume essentiellement à pouvoir les déshabiller et jouir de leur corps. Se libérer, comme femme, c'est reconnaitre et exprimer enfin le plaisir que l'on peut prendre dans une sexualité affranchie des représentations traditionnelles qui donnent à l'homme le rôle principal, quitte à jouer à la «femme objet», ce qui écarte immédiatement Bagatelle du discours féministe. C'est pourquoi on peut dire que Bagatelle imite le langage revendicatif des revues féministes plutôt qu'il ne défend une cause proprement féministe ${ }^{10}$.

\footnotetext{
9. Au plus fort des revendications féministes des années 1970, les journaux féministes obligèrent la presse magazine féminine à s'adapter aux nouvelles exigences sociales des femmes, pour éviter une importante érosion des ventes. Ainsi, les magazines haut de gamme, à l'exemple de Marie Claire et de Elle, ouvrent un espace d'expression aux thèses et aux idées féministes... tout en continuant de développer leur espace publicitaire. Cette politique prendra fin vers 1982, date à laquelle les responsables de ces titres décident de la réconciliation entre le féminisme et ce qu'ils appellent la «féminité ». Parallèlement, les journaux féministes quittent la scène, et ce, définitivement: Sorcières, Choisir, Femmes travailleuses en Lutte et les huit titres militants (Questions féministes, Histoires d'Elles, Les Cahiers du Féminisme, etc.) parus entre 1977 et 1978 s'arrêtent.

10. On remarque cependant quelques allusions plus «militantes», comme un «billet d'humeur » qui critique l'instauration de la « Journée des femmes », mais avec cet argument : «Précisons une toute petite chose: Marie Curie au Panthéon, c'est bien gentil. Mais la journée de la femme, c'est tous les jours. Et ça commence tout de suite. »
} 
«[...] Nous savons être femmes objets autant que career women, camarades ou mamans. Et c'est précisément parce que nous savons jouer tous les rôles que nous voudrions que nos hommes étendent leur registre sans nous prendre et sans les prendre trop au sérieux, d'ailleurs... Nous aimons les hommes, oui. Leur QI et leur humour, mais aussi leur nuque, leur chute de reins, leurs mollets poilus, leur épaule confortable et leur ventre, même s'il n'est pas en béton...»

Si Bagatelle feint de reprendre à son compte une tonalité féministe, sans partager les préoccupations et les objectifs politiques du féminisme, c'est que le magazine utilise ce registre pour affirmer un positionnement éditorial singulier sur le marché de la presse féminine. Bagatelle se revendique comme une presse écrite par et pour des femmes, mais ses préoccupations sont avant tout celles d'une industrie culturelle gérée à des fins commerciales ${ }^{11}$. Cette recherche de rentabilité tend à se réaliser par une gestion stratégique du double marché, qui demande d'effectuer des choix quant au public de lectrices consommatrices visé. L'emprunt d'un langage féministe ressortit donc d'une autre visée, celle de délimiter une nouvelle cible pour un magazine féminin : la « femme sensuelle » (par opposition aux femmes qui ne le seraient pas, ou, plutôt, par opposition aux autres titres de la presse féminine qui ne prennent pas en compte la part «sensuelle» des femmes). En ce sens, Bagatelle renvoie à une démarche marketing de gestion du double marché, jouant de la segmentation par l'offre éditoriale du magazine, par son discours et ses références.

11. Tout à l'opposé, les titres féministes recherchaient, eux, un public féminin plus vaste et s'inscrivaient avant tout dans le champ politique. Au XIX ${ }^{\mathrm{e}}$ siècle, on distingue deux orientations principales : le saint-simonisme à travers l'engagement de femmes comme Marie Reine Guindorf et Désirée Veret (La femme libre, 1832-1834), Eugénie Niboyet, Désirée Gay et Jeanne Deroin (La voix des femmes créé le 19 mars 1848); le courant catholique (Journal des femmes 1832-1837, La mère de famille créé en 1833) qui rappelle aux femmes leurs devoirs et leurs obligations de mère et d'épouse. Ainsi, dès ses débuts, ces journaux féministes se distinguaient précisément des journaux de mode, plus ou moins liés à une maison de couture ou à un modiste (Le miroir des Dames, Le panorama fashionable, La Capricieuse fondés entre 1836 et 1850). La presse de mode, dès ses origines (fin du XVII siècle), voit son évolution couplée à celle d'un secteur d'activité : celui de l'habillement et à l'émergence du phénomène de «mode » (sous l'Ancien Régime) alors que les journaux féministes sont affiliés à des collectifs politiques et intellectuels. La distinction «journaux de mode » versus «journaux féministes » a été proposée par SULLEROT, 1966 qui construit également un autre opposition entre ces deux catégories : la première s'adresse aux «Dames » alors que la seconde s'adresse aux «Femmes ». Cette dernière forme d'adresse finira par caractériser l'ensemble des titres de la presse féminine. Pour l'évolution de la presse féminine jusqu'à aujourd'hui, on peut se reporter à DAMIAN, 1997. 
On pourrait même envisager, plus précisément, Bagatelle comme un magazine dit de "seconde génération », dont les caractéristiques sont à la fois une spécialisation des contenus et/ou une segmentation du lectorat. Ces stratégies de positionnement s'appuient sur des «styles de vie» ou visent à s'adresser à des classes d'âge et à des catégories socioprofessionnelles délimitées ${ }^{12}$. Mais la sexualité ne peut apparaître comme un «style de vie ", ni ne peut se restreindre à une catégorie sociale ou une classe d'âge. Cette difficulté se voit dans le souci du magazine de faire exister une image de la lectrice en «femme sensuelle» au travers de sondages publiés dans chacun des numéros. Dans le numéro un, en particulier, un article («Voir enfin les hommes nus ! Pour : $53 \%$ Contre : $8,5 \% »$ ) commente les résultats d'une étude $a d$ hoc commandée à l'Institut Louis Harris. Ce sondage répond à un double objectif : évaluer l'attitude des «Françaises » vis-à-vis du spectacle de la nudité masculine et justifier l'existence de Bagatelle en transformant les réponses favorables en l'expression d'une attente à laquelle répondrait le magazine ${ }^{13}$. Cependant, préfigurant peut-être l'échec du magazine, le courrier des lecteurs publié témoigne d'une très grande variété de profils de lectrices, non seulement en termes d'âge et de modes de vie, mais également vis-à-vis de la représentation de la sexualité proposée par Bagatelle. En réalité, l'article tend plutôt à expliquer que Bagatelle cherche à s'adresser à la part sensuelle de presque toutes les femmes, ce qui l'apparente à un discours de magazine féminin généraliste et l'éloigne de la constitution d'une cible. Oser exposer au regard des lectrices des photographies d'hommes nus, même si elles sont commentées de façon libertine, ne suffit sans doute pas à assurer la pertinence d'une ligne éditoriale fondée sur la revendication d'un «droit au plaisir » féminin.

12. Les titres fondés sur les «styles de vie» adaptent les contenus de titres américains (Cosmopolitan) ou proposent des contenus inédits (à l'exemple de Santé Magazine, Médecine Douce, magazines centrés sur une information médicale plus ou moins liée au culte du corps et de l'hédonisme, ou de Vital dont Denyse Beaulieu deviendra la rédactrice en chef à la fin des années 1990). Les autres titres relèvent de la presse adolescente (20 ans, Jeune et Jolie), ou exploitent une seule image de la femme (Biba et la superwoman, Jalouse et la garce...). Ces évolutions sont par ailleurs révélatrices de l'influence des choix des annonceurs sur ce type de presse dans la mesure où ces acteurs ont intérêt à promouvoir leurs produits auprès de cibles segmentées.

13. La «femme sensuelle » telle que la construit le sondage serait plutôt jeune ( $59 \%$ de celles qui se déclarent intéressées par le fait de voir des hommes nus sont âgées de 18 à 24 ans, $56 \%$ de 25 à 34 ans), évoluerait dans un milieu économiquement favorisé (58\% de celles qui se déclarent intéressées par le fait de voir des hommes nus sont cadres supérieurs, $48 \%$ cadres moyens), profil idéal pour les annonceurs habituellement présent dans le secteur de la presse féminine. 


\section{Presse féminine et sexualité : quand Bagatelle « ouvre le bal »}

Bagatelle tente pourtant de proposer, au sein de la presse féminine, un nouvel alliage éditorial qui se confronte aux discours multiples véhiculés sur l'acte sexuel et la sexualité : ceux de la presse masculine de charme, ceux qui structurent l'imaginaire des professionnels de la presse féminine, et ceux qui sont à l'œuvre dans d'autres domaines (littérature libertine ou grivoise, sexologie, fragments «scandaleux» de la culture de masse). Nous n'aborderons pas ici les emprunts de Bagatelle à la presse masculine de charme, sauf pour dire que le magazine reprend évidemment à des titres tels que Playboy, Penthouse, etc., la présence en pages centrales du strip-tease de jeunes mannequins du sexe opposé. Mais tout distingue Bagatelle de cette presse de charme dans la mesure où le couple que la lectrice forme avec son compagnon demeure l'horizon même d'inscription des fantasmes sexuels. De fait, Bagatelle se défend d'une telle affiliation, notamment au travers de son courrier des lecteurs qui se révèle être un des lieux principaux de justification de son existence: si Bagatelle revendique une forme de levée des «inhibitions » féminines, il écarte aussi par ce biais pornographie et vulgarité. La publication de lettres de lectrices permet au titre de tenir un équilibre entre les critiques qui lui reprochent de ne pas aller assez loin (nombre de photos d'hommes nus trop réduit) et celles qui qualifient certains choix du journal de «vulgaires » en assimilant le journal aux magazines de charme masculins.

Cet équilibre n'est viable que si le magazine se tient au carrefour de frontières multiples et élabore des définitions parcellaires, multiples et parfois contradictoires de la féminité. Il partage, de fait, cette position d'ambivalence avec les autres titres de la presse féminine généraliste. Nous pouvons peut-être expliquer celle-ci par la difficulté qu'éprouvent les acteurs eux-mêmes à proposer des représentations claires de «la femme » en termes d' «identité féminine », les discours oscillant entre une appréhension biologique du «féminin» et une définition sociale et économique de la «femme»d'aujourd'hui ${ }^{14}$. Cette difficulté est perceptible dans la façon

14. DAMIAN, 1995. Leurs discours, leurs croyances mêlent, de façon inextricable, deux types de représentations. La première considère "la» féminité comme une «entité naturelle », innée, irréductible à l'histoire et liée à un état biologique. La «nature féminine », universelle, renvoie alors à des caractéristiques partagées par l'ensemble des femmes quel que soit leur milieu social, véritable «communauté » dont le langage et le rapport au corps échapperaient largement aux hommes. La seconde se fonde sur une interprétation des rôles, des tâches et des fonctions actuels des femmes au sein de la société. Cette «personnalité 
dont ils justifient l'existence de la presse féminine généraliste : pour les acteurs de ce secteur, celle-ci s'appuierait sur une sociabilité extérieure au média lui-même et spécifique aux femmes, parce qu'elle serait liée à des valeurs affectives, psychologiques mais aussi à des données sociales et économiques. Dans le cas de Bagatelle, les échanges libertins sur la sexualité entre «copines » constituent de façon explicite pour les rédacteurs le modèle de référence à reproduire dans les colonnes du magazine, comme nous le verrons un peu plus loin. Ainsi, ce journal est à la recherche d'une communauté de lecteurs fondée sur une certaine conception de la sexualité, qui n'exclut donc pas la lecture du magazine par des hommes. Certaines lettres et articles incitent, et invitent même les lectrices à partager la lecture du magazine avec leur compagnon. En ce sens, même s'il cherche à favoriser l'expression d'un discours sur le plaisir féminin, Bagatelle se distingue du discours féministe des années 1970 qui tentait d'élaborer (voire de conceptualiser) une «identité sexuelle» propre à la «femme» en promouvant une parole qui serait véritablement (voire exclusivement) celles des femmes sur cette question. Bagatelle, lui, pour construire un projet éditorial original au sein de la presse féminine, semble davantage se préoccuper de limiter les rejets et les exclusions que peuvent induire ses choix rédactionnels, en témoignant à la fois d'une prudence axiologique (aucune pratique n'est a priori condamnable), et d'un désir d'ouverture en direction des hommes.

«Ces hommes-là [la rédactrice vient d'évoquer quelques stars du cinéma et de l'audiovisuel] ont en commun de n'être jamais là où on les attend. Virils aucun doute là dessus - mais d'une douceur parfois féminine... Désirables, mais capables d'élans presque mystiques [sic]. Limite hard, mais au fond sentimentaux... Multiples, quoi. Comme les femmes. »

sociale » propose l'image d'une femme «multifacette», sorte de «super-woman » capable d'assumer des responsabilités professionnelles et familiales, d'associer féminité et émancipation, grâce à une prodigieuse capacité d'organisation. Par son mode de vie, elle incarnerait l'ensemble des droits acquis par les femmes au cours de ces trente dernières années, et démontrerait que «féminité » n'est pas incompatible avec féminisme. Il faut pondérer toutefois les discours tenus par ces responsables sur la (les) femme(s) et la presse féminine généraliste en les confrontant aux contraintes de production propres à ce type de presse. En fonction des choix marketing, et donc du «cœur de cible » auquel s'adresse un magazine support, les professionnels favoriseront dans leurs productions symboliques tel ou tel type de représentation et telle ou telle fonction éditoriale. 


\section{De la posture des journalistes comme stratégie de justification du titre}

A travers la question du «droit au plaisir» et de celle de l' «identité » féminine, on voit que sont à l'œuvre dans le discours de Bagatelle de multiples tensions, même si l'on note la recherche d'équilibres dans l'élaboration des contenus rédactionnels. De ce point de vue, le rôle et la place accordée à la figure de la rédactrice en chef (Denyse Beaulieu) prend tout son sens car il permet d'assurer plus qu'une cohérence énonciative tout au long du magazine. Pour un(e) journaliste, en effet, écrire sur la sexualité de façon «libre » ne peut pas être anodin: l'image de soi morale ou professionnelle se joue tout particulièrement sur une question de ce genre, qui impose tout à la fois un ton «léger» (pour ne pas trahir des inhibitions, par exemple) mais aussi une certaine décence (puisqu'il s'agit d'une parole ou d'un écrit public). La question se complique lorsqu'il s'agit d'écrire au sein d'un magazine qui se veut explicitement porté sur la sexualité. En effet, une énonciation qui apparaîtrait «lointaine » (sans engagement supposé de la part de celui ou celle qui écrit) ruinerait le projet même du magazine qui est d'entrer en contact, de nouer une relation avec une communauté de femmes dont la sensualité serait affirmée, voire revendiquée.

En sens inverse, une énonciation impudique ou indécente (ou simplement dévoilant des affects ou des fantaisies sexuelles qui ne concernent que celui ou celle qui écrit), ou une «liberté » de langage trop marquée sur les questions sexuelles (conduisant à une forme de vulgarité) risquerait de choquer et de menacer l'image morale et professionnelle du rédacteur. C'est pourquoi, comme on va le voir, la relation énonciative que propose le journal a précisément pour but de construire un espace imaginaire inédit (afin de produire la différenciation nécessaire à sa viabilité sociale et économique) qui accueille la lectrice au sein d'un groupe de «copinescoquines » (neuf articles sur dix sont signés par des femmes dans Bagatelle), groupe qu'elle peut reproduire elle-même au sein d'un acte de lecture collectif (attesté dans plusieurs lettres). Cette solution énonciative permet ainsi d'utiliser l'interlocution « entre copines » comme un modèle de réglage de la relation entre rédactrice et lectrice, sur le mode d'un échange d'expériences (et de «trucs»). Cette relation suppose, en effet, à la fois une dimension publique limitant en principe les débordements de l'intime à ce qui est partageable entre paires (à la différence de la relation exclusive à une confidente, ou à la différence d'une confession, par exemple) et une certaine connivence au sein de laquelle l'humour, les allusions, voire le maintien d'un certain secret, jouent un rôle primordial. 
En ce sens, Bagatelle peut reprendre sans peine le code d'une énonciation relativement marquée, voire très marquée (Marcelle Ségal dans Elle, par exemple), qui prédomine dans la presse féminine (surtout haut de gamme) à partir des années 1960. Le magazine peut exploiter tous les ressorts que permet ce mode énonciatif, et tout particulièrement le passage permanent du «nous» au «je», au nom d'une communauté d'expériences ou de sentiments partagés - ici «sensuels »-propres aux «femmes » (alors même qu'il s'agit très souvent des sentiments ou des expériences propres à un certain type de femmes qui achètent tel magazine particulier). De plus, pour éviter un discours qui parlerait sans aucun relief de sexualité, la rédactrice va pouvoir précisément utiliser ce «nous» comme un masque commode pour engager dans son énonciation une émotivité propre à réveiller en chaque lectrice certains émois liés à la sexualité, tout en cachant les siens propres. Pour étudier ce travail d'expression des journalistes, il nous paraît alors pertinent de distinguer en analyse de discours la légitimité, qui détermine en amont le statut (institutionnel, organisationnel, etc.) du locuteur, de la crédibilité, qui a trait à l'action même du locuteur et dépend non seulement du statut de celui qui s'exprime, mais peut aussi se conquérir, ou se perdre, dans l'acte même du discours, ce dont atteste l'analyse de l'ethos (c'est-à-dire l'analyse de la préservation ou la modification des images de soi de celui qui parle ou qui écrit ${ }^{15}$ ). En effet, par delà les exemples de magazines féminins libertins à l'étranger ou les « changements » de mentalité qui servent à Bagatelle à assurer sa raison sociale, ou légitimité, l'enjeu pour les rédactrices est alors de construire en acte la crédibilité de chaque article et de chaque signature afin de faire la démonstration de la pertinence de la ligne éditoriale choisie, numéro après numéro. Dès lors, de même que l'on peut voir comment une crédibilité peut construire ou défaire une légitimité, on observera comment il est possible, à la fois, de distinguer et de lier ethos personnel (définition rhétorique) et ethos social ou professionnel (définition sociologique) en étudiant les répercussions de l'un sur l'autre.

15. SOULEZ, 1998, p. 399-409. L'étude évoquée ici a porté sur une série de cas mettant en jeu l'identité professionnelle de journalistes de télévision. Ainsi, par exemple, en pleine affaire des «animateurs-producteurs » (1996), alors qu'il était la cible principale des critiques, J.-L. Delarue s'est efforcé de restaurer sa crédibilité au sein même des émissions qu'il animait (ethos personnel), faisant du même coup la démonstration en acte de sa capacité d'animateur au cours d'un moment difficile (ethos professionnel). 
On peut considérer que trois éléments constitutifs caractérisent le travail sur l'ethos en jeu dans Bagatelle dans ce cadre : l'engagement très singulier de sa rédactrice en chef, Denyse Beaulieu, la construction d'une figure de rédactrice directe, experte mais moralement irréprochable, mais aussi d'une relation entre rédactrices et lectrices à travers une expressivité «spontanée » (éclats, points d'exclamation, soupirs figurés par des points de suspension). Si l'on s'attarde un instant sur le premier point, qui est le plus original, on constate que l'importance de l'espace rédactionnel occupé par la rédactrice en chef identifie d'emblée, et très fortement, le journal à Denyse Beaulieu. Il ne s'agit pas simplement du «je » traditionnel des éditoriaux de magazines féminins, à travers lequel la rédactrice en chef (ou l'éditorialiste) se fait, en réalité, simple porte-parole d'un «nous » représentatif des heurs et malheurs féminins, mais véritablement d'une sorte de construction de la personnalité de D. Beaulieu en démiurge: elle est celle qui peut offrir aux femmes comme un cadeau ce qu'elles attendaient depuis si longtemps. La fragilité de cette raison d'être du journal (même s'il se mue une raison sociale) est donc cela même qui fait sa crédibilité, et qui autorise du même coup cette omniprésence et omnipotence d'un «Je, Denyse Beaulieu». Par là, en un lieu stratégique comme le «paratexte» que constitue l'éditorial ${ }^{16}$, la rédactrice se construit comme source principale et réelle garante de la ligne du magazine, comme l'« auteur » du magazine si l'on suit la définition qu'en propose François Jost : «celui qui accroît la confiance en ses textes en les relayant à travers les divers épitextes et paratextes ${ }^{17}$ ».

Denyse Beaulieu met toute sa personnalité dans la balance, comme pour conjurer toute difficulté à oser enfin parler de sexualité féminine, liant le journal à son destin personnel :

«Vous vouliez un magazine féminin qui vous parle de ce qui vous importe le plus : l'amour, les hommes... bref, la vie. Et qui vous en parle comme on le fait aujourd'hui entre nanas, avec franchise et humour. C'est vous qui me le

16. Outre qu'il constitue le premier texte important du journal issu de la rédaction, ou qu'il présente le dossier du mois, l'éditorial joue le rôle dévolu traditionnellement aux préfaces (ou aux autres formes de «paratexte ») en étant le lieu où s'élabore un discours sur le journal dans son ensemble.

17. Jost explique quelques pages plus tôt : «Avant de renvoyer à autorité, 'auteur' signifie d'abord 'celui qui accroît' (d'augere, 'augmenter') et plus spécifiquement celui qui accroît la confiance, le crédit, à savoir le vendeur. Ce n'est pas du côté de la métaphysique qu'il faut chercher la justification de cette instance, mais bien plus du côté de l'économie. » JOST, 1992 p. 58 et 87. 
dites ; c'est ce dont j'avais envie, en créant Bagatelle. Après tout, pourquoi les femmes n'auraient-elles pas droit à un journal qui traite de sexualité sans hypocrisie et montre les beaux mecs sans feuille de vigne? »

Outre cet ancrage à l'«envie» affichée d'une femme véritablement «entreprenante», on peut remarquer que l'ethos de Denyse Beaulieu contribue à dessiner le cadre général au sein duquel vont pouvoir s'inscrire les autres discours, en mobilisant à la première personne un garant «moral » pour la sexualité presque inattendu, le mariage, accompagné de la revendication d'une sexualité "à la limite», la sodomie. En effet, D. Beaulieu revendique haut et fort le fait d'être mariée, ce que l'on voit par exemple dans l'éditorial («Youpiii, on se marie !») qui annonce le dossier du numéro consacré au mariage. Elle mêle si bien sa vie de femme et d'épouse à celle du journal que celui-ci devient tout naturellement la tribune d'une adresse directe fictive à son mari ! A l'autre bout du spectre de la «moralité sexuelle», D. Beaulieu propose une nouvelle érotique dont l'intrigue mène l'héroïne (qui parle à la première personne) à une sodomie à demi-consentie avec un torero mort-vivant. Touchant à un classique de l'érotisme (l'amour avec un mort) qui permet ici d'afficher par son excès un caractère non réel, Bagatelle franchit donc, à nouveau avec l'aide de sa rédactrice en chef, sous couvert de «fiction» assumée à la première personne, une frontière possible de la décence. Une fois de plus, le destin du journal est lié à sa rédactrice en chef. La fiction permet de préserver l'ethos mais la lectrice est incitée à lire le texte comme le lieu de la révélation des «fantasmes » de la rédactrice en chef. Par ce geste, en réalité, Denyse Beaulieu montre, sans danger, jusqu'où elle peut aller, ce qui finalement est censé renforcer sa crédibilité tout à la fois de femme "libérée » et de rédactrice en chef (son talent d'écriture).

La mise en jeu de l'ethos de Denyse Beaulieu dans le magazine a donc une fonction importante dans la mesure où elle permet de mesurer les frontières tant du rapport à la loi sociale que du rapport aux «fantasmes » que propose Bagatelle, mais elle ne constitue sans doute pas une condition suffisante de viabilité pour le magazine. Face à la revendication d'un droit au plaisir, et la difficulté à définir un profil féminin «sensuel», la focalisation sur la rédactrice en chef peut être lue, à l'inverse, comme le symptôme d'une segmentation qui ne parvient pas à se constituer comme telle. C'est alors peut-être du côté de la production de récits susceptibles d'être partagés, plutôt que de l'identification stricto sensu à une figure initiatrice, que peut se faire l'adhésion au projet du magazine, comme le suggère implicitement 
l'invitation faite aux lectrices de prendre la plume à leur tour : "Bagatelle ouvre désormais ses pages 'fiction' aux femmes dotées d'une jolie plume, qu'elles soient écrivains reconnues ou débutantes douées. Je me suis permis d'ouvrir le bal : mais j'attends de vos nouvelles. »

\section{BAGATELLE : FRAGMENTS D'UN DISCOURS SEXUEL AU SEIN DE LA PRESSE FEMININE?}

Si la lectrice ne prend pas la plume, on peut néanmoins supposer qu'elle s'investit fortement dans les récits proposés par le magazine, puisque c'est la réorganisation de la place de chacun qui est l'objet de toutes les attentions, et non l'originalité des figures de l'amour utilisées (l'homme qui « fait fantasmer » est souvent un «latin lover»; l'Orient et l'Inde - «danse du ventre », hammam et kamasutra obligent - sont des lieux inépuisables d'inspiration, etc.). Bagatelle joue ici avec les normes pour partager avec ses lectrices cette manière «libérée » de vivre la sexualité féminine, affranchie des canons traditionnels du désir féminin (secret, patience, voire souffrance, caractère dissymétrique, etc.). Par quel moyen le magazine peut-il élaborer un discours à la fois pertinent et crédible, alors que ce choix éditorial interroge directement les normes auxquelles doivent généralement se conformer les femmes, et plus particulièrement les modèles de conduite liées à la sexualité ?

Pour rendre compte des écarts possibles du magazine par rapport à ces canons, nous nous inspirons de l'approche de John Gagnon et William Simon qui considèrent les normes qui prédominent dans la sexualité comme le produit d'une «imprégnation par des récits impliquant des séquences d'événement », plutôt que comme le résultat d'une inculcation de règles et d'interdits ${ }^{18}$. Pour Gagnon et Simon, «la conduite sexuelle doit être considérée comme une scripted behavior ${ }^{19}$ ». Un «script » est un «schéma cognitif bien structuré », un «scénario ${ }^{20}$ » (telle la série d'actions qui

\footnotetext{
18. BOZON et GIANI, 1999, p. 70.

19. Ibid.

20. Pour Gagnon et Simon : le script peut prendre une forme assez générale (la série virginitémariage-filiation par exemple) ou s'incarner au sein d'interactions quotidiennes (du baiser à la caresse, etc.). Le premier script est dit «culturel», le second «interpersonnel». Un troisième niveau de script, les scénarios «intrapsychiques », plus difficile à atteindre, relève de la vie intérieure des individus et permet, à partir « d'éléments symboliques fragmentaires, [de] scénarios culturels plus largement partagés, [et d'] éléments d'expérience personnelle »,
} 
conduit d'un rendez-vous au cinéma jusqu'à la chambre à coucher). Puisque nous ne connaissons pas les pratiques qui les accompagnent, les récits que propose Bagatelle ne sont alors que la forme manifeste de schémas cognitifs, même s'ils sont expressément tournés vers la réalisation d'actions. Nous analyserons donc un discours sur les conduites sexuelles et, plus précisément, des propositions de conduites dont la légitimité reste à construire, du point de vue de l'individu (la lectrice et son histoire personnelle) et/ou de la société (le rapport de ces conduites aux canons).

Pour prendre de la distance par rapport aux conduites sexuelles habituelles, telles qu'elles se présentent en particulier dans le reste de la presse féminine, Bagatelle est, en effet, contraint d'inventer (ou de réinventer) de nouvelles situations qui mènent à l'acte sexuel, de changer les rôles convenus, de produire de nouvelles figures d'identification liées à la sexualité. Nous ne pouvons pas considérer que tel récit «novateur» proposés par Bagatelle correspond à tel schéma cognitif préalablement fixé, puisque ce caractère même d'innovation écarte les conduites type, et nous avons plutôt affaire à l'élaboration en cours de l'un de ces scénarios, c'està-dire au rôle de l'imagination dans la production des scripts sociaux de la sexualité. On peut donc observer que les médias ne sont pas seulement des lieux où s'énoncent les conduites et les interdits ${ }^{21}$, mais aussi des laboratoires de normes, fonction entrevue par exemple par Maigret à propos des identités sexuelles des adolescents ${ }^{22}$. C'est ainsi que Bagatelle va remettre en cause implicitement «le scénario dominant qui prévoit que c'est à l'homme de faire les propositions ${ }^{23}$ », pour proposer à la jeune femme de prendre l'initiative. En ce sens, Bagatelle donne corps, au moins sur un plan imaginaire, à une critique qui s'était énoncée depuis longtemps dans la presse féministe et même féminine.

une forme de « coordin[ation] entre la vie mentale et le comportement social, [en] opér[ant] la reconnaissance des situations sexuelles ». BOZON, GIAMI, 1999, p. 70.

21. GAGNON, 1999, p. 74-75.

22. MAIGRET, 1995.

23. BOZON, GIANI, 1999, p. 71. 


\section{«Un parfum d'aventure » : l'initiative féminine au cour des scénarios sexuels de Bagatelle}

A un premier niveau ${ }^{24}$, et suivant son slogan («le magazine féminin qui déshabille les hommes »), le discours dominant de Bagatelle est de faire l'éloge de l'initiative féminine. Photographies, récits, lettres, conseils promeuvent un rôle actif de la femme dans les scénarios sexuels, qu'elle soit «fidèle » ou "adultère », comme on le voit dans un long article-manifeste du premier numéro. Bagatelle propose des actions et des rôles féminins qui transforment le déroulement des scénarios habituels et relance potentiellement le désir féminin : l'initiative féminine est présentée comme la clé sine qua non d'un changement. La critique des scénarios classiques est menée au nom de l'ennui ou de la souffrance qu'ils entraînent chez la (jeune) femme. Dans le premier cas, l'homme apparaît comme une force d'inertie, tandis que l'action féminine sauve le couple de la monotonie ${ }^{25}$, dans le second, la femme doit se hisser au même degré d'initiative et d'action que l'homme adultère afin de sortir d'une forme de passivité douloureuse ${ }^{26}$. La norme de l'égalité entre les sexes sert ici de garant aux nouveaux récits, mais on peut se demander si, comme on le verra, cette référence ne révèle pas une difficulté à concevoir de nouveaux rôles féminins «actifs » qui ne soient pas calqués sur ceux des rôles traditionnels du séducteur masculin.

La monogamie au long cours importe plus aux femmes qu'aux hommes, et c'est à elles qu'il revient d'insuffler une vitalité toujours renouvelée à leur relation amoureuse. Il faut bien que quelqu'un s'en charge, et la meilleure façon de mener la tâche à bien, c'est évidemment de l'assurer soi-même.

On le voit d'emblée lorsque le magazine suggère ainsi à ses lectrices d'investir certains lieux de «drague», comme les bars où l'on peut

24. Il s'agit du niveau «interpersonnel » des scénarios pour Gagnon et Simon (voir note précédente).

25. De façon générale, ces scénarios supposent la levée d'inhibitions fortes qui sont liées à l'identification de certaines pratiques (fellation, par exemple), ou postures (la domination), à celles de «professionnelles » et non de conjointes.

26. Le passage suivant est ainsi une sorte de métaréflexion sur les schémas cognitifs euxmêmes en jeu dans l'adultère : «Une femme qui prévoit un cinq-à-sept pour jeudi après-midi y pense dès le lundi matin. L'énergie mentale qu'elle y consacre tend à brouiller dangereusement ses autres priorités. Comme elle en est consciente, elle se sent coupable, perd son naturel avec son mari et se dirige tout droit vers le désastre. » 
retrouver des top-models masculins. Par là, inversant les rôles, l'homme devient très souvent, dans les scénarios de Bagatelle, la proie des envies féminines. Plus avant dans le script du désir, Bagatelle propose de nombreux récits (fictionnels ou supposé vécus), ou des scènes (photos de mode, strip-teases), qui font de la femme la protagoniste principale, ou, au moins, la coactrice du scénario sexuel. L'homme devient alors, parfois explicitement, un «objet». L'analyse d'un roman-photo du $n^{\circ} 3$ («Tu m'attends... surpris par l'objectif, seul dans un palace ») est révélatrice de ce point de vue. Dans une chambre d'hôtel, un jeune homme se déshabille progressivement, va prendre un bain, puis s'installe en peignoir une coupe à la main avant, semble-t-il, d'accueillir la femme qui le faisait attendre, et qui, telle la lectrice, demeure hors-champ. Accompagnant pas à pas le mouvement du jeune homme, une forme de voix over qui dit «je», à laquelle la lectrice peut s'identifier, donne sens à la scène, lui sert de légende ${ }^{27}$. Par là, l'initiative féminine est valorisée parce qu'elle produit un véritable spectacle. Bagatelle produit ainsi l'atmosphère favorable à une sexualité «libérée» par un travail de mise en pages et de mise en récit élaborée qui vise à intégrer la lectrice ${ }^{28}$.

\section{Comment dire, comment lire : je désire ?}

«A vos fantasmes... », écrit parfois le journal : Bagatelle se conçoit donc d'abord comme un lieu de projection des fantasmes féminins, un activateur de scénarios «coquins» qui peuvent nourrir l'imaginaire de la lectrice, avant, peut-être, de lui «donner des idées » pour ses scénarios quotidiens. On peut se risquer à quelques hypothèses sur la manière dont Bagatelle conçoit la projection de ses lectrices dans les récits qu'il propose. L'analyse des places énonciatives proposée par Eliséo $\operatorname{Veron}^{29}$ prend ici le relais de l'étude des scripts parce qu'elle permet d'interpréter le rôle supposé des

27. Cette voix semble s'adresser à lui (sur le modèle des jeux à énigmes où l'on dit «Tu brûles...»), par exemple: "Glisse ton corps dans l'eau chaude... Abandonne-toi à sa caresse, oublie tout ce qui n'est pas nous. »

28. Pour l'analyse de ce roman-photo, et en particulier du statut du lieu d'où parle l'énonciateur, nous nous sommes appuyés sur SOULEZ, 1999.

29. VERON, 1984. Comme on le sait, E. Veron propose d'étudier l'énonciation comme un système de places qui constitue un circuit interprértatif (le « contrat de lecture ») fondé sur un réglage réciproque de l'énonciateur et du destinataire du discours. Ainsi, le destinataire peut «reconnaître » ou non la place que le locuteur (qui représente un journal) lui indique dans son énonciation (le dire - et non le dit) par exemple lorsque celui-ci lui propose une relation pédagogique ou fondée sur une connivence culturelle, etc. 
relations entre énonciateurs dans ces fragments de scénarios qui peuvent nourrir les scripts intérieurs de la lectrice (appelés «intrapsychiques » par Gagnon et Simon). Le magazine propose, en effet, à la lectrice d'investir différents «je », c'est-à-dire différentes places subjectives pour se situer dans ces récits reformulés du désir. Le rôle de la première personne se voit de façon exemplaire dans l'article «Mes dix résolutions pour une année sensuelle » proposées à la lectrice dans le numéro deux. L'identification à un «je » féminin qui ose et affirme son désir est l'une des clés du discours de Bagatelle sur la sexualité féminine: ce «je»plein d'audaces et de ressources est proprement l'alter ego du «vous » que le magazine ne cesse d'inciter à l'action (sinon à la débauche). Il est donc une figure discursive du « vous » qui passe (enfin) à l'acte. A toutes les étapes du script de la relation sexuelle, sont donc valorisées les conduites dites « actives » de la femme, du flirt $^{30}$ à la recherche de nouvelles positions au sein de l'acte sexuel, en passant par le premier rendez-vous.

Mes «Mes dix résolutions pour une année sensuelle:

(...) Résolution 4 : J'ai droit à l'égoüsme

(...) Résolution 7 : Je cultive mes fantasmes, même les plus cochons

Résolution 8: Je suis mes humeurs

Résolution 9: Je ne le lâche pas après l'amour

Résolution 10 : J'ai le droit d'être une salope. »

Bagatelle complexifie ainsi le système énonciatif de la presse féminine en proposant un jeu à quatre rôles: la journaliste incitative ${ }^{31}$, une instance engagée dans une sexualité active qui dit «je» (une lectrice qui écrit ou envoie une photo, un personnage qui raconte, ou une personnalité qui témoigne), la lectrice, et son amant/compagnon. De ce point de vue, le rôle de stars «proches» (telles Sapho, Amanda Lear, ou «Mademoiselle Agnès » de Canal+), souvent liées au petit écran ${ }^{32}$, est celui, essentiel, de médiatrices qui permettent de (se) dire ouvertement : "Je désire, je veux cet homme. » «Pulpeuse », « sexy », «baby-vamp», elles proposent ainsi un

30. Quelques titres l'indiquent assez bien, qui utilisent l'impératif : «Osez le flirt» $\left(\mathrm{n}^{\circ} 2\right)$, «Réveillez votre énergie sexuelle », $\left(\mathrm{n}^{\circ} 4\right)$, «Décodez-le dès la première soirée », $\left(\mathrm{n}^{\circ} 3\right)$ «Faites de votre homme un super coup $»\left(\mathrm{n}^{\circ} 1\right)$.

31. «Osez! » (formule récurrente, en couverture du $n^{\circ} 2$ ) ; «Faites zig-zig en musique »; «Jouez les prolongations avec ce balconnet à 'coussinets valorisants' Triumph (puisqu'on vous le dit) » (légende d'une photo, etc.).

32. CHALVON, PASQUIER, 1990. 
regard mi-expert, mi-spontané sur le corps des «pin-ups» masculins que dévoile le magazine.

«Ah, celui-là ! Qu'est-ce qu'il est sexy ! C'est too much. Aimerais-tu qu'il me prenne dans ses beaux bras musclés? Il faut bien avouer qu'il dégage une certaine puissance $[\ldots]$ Est-ce que je me le ferais ? »

Chanteuse Sapho, commentant une série de photos en « voix-over »

La lectrice, quant à elle, consent à se libérer, à se projeter, à jouer le jeu de cette place énonciative qu'on lui propose : «Après avoir lu [Bagatelle] en détail et avec attention, je me suis sentie beaucoup plus libre par rapport au sexe. [...] Je voudrais aussi ajouter que la 'pin-up' mec du numéro 3 m'a fait fantasmer » [lettre]. De ce point de vue, tout en valorisant une parole féminine orientée vers la sexualité, une parole projective, Bagatelle occupe, en réalité, une place masculine dans l'organisation discursive du désir féminin, celle de déclencheur, tel le rôle traditionnellement dévolu au Prince Charmant des contes, censé éveiller en chaque lectrice le désir qui sommeillait jusque là. La Belle peut alors enfin exulter : "Telle Lazare dans son sépulcre, je pourrissais, rongée de complexes en tout genres, écrasée de diktats péremptoires [...] et puis est paru Bagatelle. 'Lève-toi et marche !' » [lettre, $\mathrm{n}^{\circ} 2$ ]. Le magazine fait donc logiquement l'objet d'investissements affectifs croisés au sein de groupes, surtout féminins, tel un objet sexuel que l'on s'échangerait entre amies : « Je prête votre journal à des ami(e)s et nous nous amusons bien. »; "J'ai immédiatement prêté le magazine aux copines et sauf quelques mijaurées, elles se sont toutes régalées » [lettre]. Mais, bien souvent, cette sensualité des récits, ces "photos fantasmantes", ont finalement pour objectif de modifier très concrètement la conduite sexuelle de la lectrice avec «son» homme, qui est donc bien partie prenante du discours, en tant qu'il est ce qui oriente le discours, sa finalité.

«C'est l'homme que l'on veut pour qu'il nous veuille aussi !»

«Faites de votre homme un bon coup », on l'a lu ensemble sous la couette, en riant, en jouant, en testant !»

C'est pourquoi, on peut dire que si Bagatelle évoque des scénarios non traditionnels (adultère, amour à plusieurs ${ }^{33}$ ), ou rarement rendus public dans la presse féminine de l'époque (situations libertines, sexe oral) - et se refuse

33. «Le fantasme du triolisme est une façon de mettre en jeu une sensualité intense qui n'ose s'exprimer. » Réponse d'une «psychologue » à une lettre. 
bien sûr à les condamner, ceux-ci constituent davantage des modèles imaginaires pour une sexualité «active » qu'un ensemble de conduites à suivre dans la vie réelle. De façon générale, et pour ne pas verser dans la «presse spécialisée», mais prendre sa place parmi les autres magazines féminins, Bagatelle défend souvent l'idée qu'une relation de couple active et harmonieuse permet d'allier érotisme et amour, à l'instar de l'éloge du mariage par sa rédactrice en chef. On peut mesurer ainsi le poids des normes morales à travers les conventions narratives, et voir jusqu'où le magazine peut jouer avec elles.

\section{La production normative de Bagatelle : l'alcôve et la couette}

Rencontrant la question du couple, horizon quasiment indépassable de la presse féminine, le magazine est face à une contradiction difficile à surmonter : donner le goût de l'aventure et de l'imprévu tout en respectant la loi d'airain d'une sexualité qui s'épanouisse dans le cadre de la monogamie traditionnelle. Bagatelle tente alors une forme de synthèse en jouant sur les limites de ce cadre, mais sans effacer la tension entre un discours libertin, potentiellement hétérodoxe, et un discours sexologique à visée explicitement pratique dans le cadre d'un couple établi. Se dessine ainsi le projet une cartographie du désir orthodoxe (le couple hétérosexuel stable) jalonnée cependant de chemins de traverse, de nouvelles perspectives ou d'imprévus, qui, en jouant avec les limites (sociales ou intimes) de la relation, rendent le paysage plus attrayant (d'où la valorisation du sexe oral, par exemple). En termes de scripts, on peut donc dire que, lorsque Bagatelle se confronte à des scénarios culturels hétérodoxes, comme l'adultère, c'est pour en retirer quelques éléments qui vont renouveler les scripts interpersonnels, et non pour abandonner le script dominant (culturel) du couple classique ${ }^{34}$. En témoigne, dans le sommaire du $\mathrm{n}^{\circ} 1$, cet appel à tromper son compagnon qui n'est qu'une manière de rejouer la sexualité dans le couple : «Trompez-le ! Pendant que vous en avez envie (avant d'en avoir besoin). Ou bien restez fidèles, mais sachez les règles du jeu ! » Dans le numéro suivant, Bagatelle prend soin de publier la lettre d'un lecteur (la seule lettre d'homme publiée) qui le comprend bien ainsi : "Mais à regarder de plus près, votre article est loin de faire l'apologie de l'adultère, et vos conseils dignes d'être pris en considération. » Bagatelle propose donc en quelque sorte un rafraîchissement des scripts sexuels, mais ne sort pas du cadre normatif du

34. Pour les notions de «script culturel » et de «script interpersonnel », voir la note 16. 
couple hétérosexuel stable : il vise, à partir de fragments culturels plus ou moins orthodoxes qui alimentent le désir de la lectrice, à normaliser des imaginaires «marginaux », «scandaleux » au sein du scénario classique. Observons de plus près comment se joue ce processus de normalisation.

Le travail normatif de Bagatelle se voit nettement dans son inventivité lexicale, celle-ci témoigne, en effet, de façon très intéressante des tensions entre la proclamation de fantasmes et désirs féminins «à la limite » et leur inscription dans la réalité quotidienne du couple. Le langage amoureux vient souvent à la rescousse pour sublimer les élans les plus «sauvage[s]», comme l'atteste, par exemple, la formule « étreinte-bitume » pour désigner le corps à corps impulsif des amants dans l'ombre d'une porte cochère. On peut considérer que cohabitent deux formes principales de «transcription»: l'une recycle au sein de la vie sexuelle domestique l'univers du noceur masculin, l'autre traduit en langage sexologique les transports amoureux, comme si l'important était, au bout du compte, le « résultat» obtenu. Ceci nous montre qu'il y a une tension entre la capacité des scénarios à faire «fantasmer» la lectrice et un discours à visée pratique qui risque de transformer l'acte sexuel en simple « recette » sans saveur.

Le premier type de discours vise à «domestiquer» des figures «scandaleuses » (titre d'une rubrique) comme les vamps, les strip-teaseuses, les grandes séductrices pour nourrir des scénarios «coquins» de façon fragmentaire sans pour autant menacer le récit principal que se propose une lectrice supposée attachée à une sexualité dans le cadre d'un couple stable. Il s'agit simplement de s'inspirer de ces figures pour construire une dramaturgie érotique à deux («Comme Kim Basinger, apprenons à le vamper»). On peut remarquer une forme de récupération de la tolérance autrefois consentie aux hommes pour les divertissements en dehors du foyer (parce qu'ils permettaient une forme de «défoulement» et évitaient l'adultère ou le recours à la prostitution), au profit de conduites féminines «actives» habilement transférées dans l'espace domestique. Plus généralement, s'il contribue à un affaiblissement des frontières sexuelles (masculin/féminin) ou sociales (moindre condamnation de la sexualité comme divertissement), ce discours de la réversibilité des rôles a pour effet paradoxal de maintenir fermement le couple comme cadre de jeu, et de proposer simplement une redistribution des rôles.

Dès lors, l'enjeu se déplace: il ne s'agit plus de valoriser une femme « sensuelle », «libérée » ou conquérante, mais de favoriser une plus grande 
« harmonie» sexuelle au sein du couple. Ce qui passe, en effet, dans les «théories » sexologiques (du type Masters \& Johnson) par une plus grande participation de la femme, mais, surtout, par une meilleure connaissance du corps de l'autre (en l'occurrence l'homme). Dans cette perspective, qui voit la sexualité comme une simple pratique plus ou moins réussie (validée par la jouissance des deux partenaires), la limite à franchir n'est plus celle de l'acte illégitime (l'adultère) mais simplement du silence, de la fausse pudeur. Le rôle de la femme dans le couple suppose alors un certain nombre de compétences comme celles de savoir user de séduction, d'audace ou de ruse, ou comme celle, surtout, d'être à même de métamorphoser en douceur le compagnon 'ordinaire' en amant extraordinaire (la jeune femme occupe alors un rôle d'éducatrice). Outre les références explicites aux textes canoniques de la sexologie, les termes techniques et les mots consacrés (comme «partenaire ») se retrouvent au milieu du bric-à-brac «libertin » du magazine. Les fantasmes ne sont dès lors plus qu'un auxiliaire pour une pratique efficace de la sexualité : «L'imagination est la plus sensible des zones érogènes », explique un article.

Bagatelle propose donc deux discours à la fois, qui ne s'articulent pas toujours : le premier, celui de l'alcôve, qui vise à différencier le magazine du reste des autres magazines féminins, tire la sexualité du côté du fantasme, du «spectacle intérieur » du désir qu'alimenteraient les différents scénarios «libertins » du journal, le second, celui de la couette, vise un décodage systématique de l'acte sexuel en éléments «réutilisables », tant pour lever des inhibitions que pour s'essayer à de nouvelles pratiques, rejoignant ainsi la tendance générale de la presse féminine qui fait du magazine un « outil de vie ». Si bien qu'il n'est pas impossible de considérer que Bagatelle creuse potentiellement l'écart entre les pratiques « réelles » et le discours qu'il tient sur elles, même si les conseils pratiques proposent une forme de traduction du «fantasme» (l'alcôve) en "réalité » sexuelle au sein du couple (la couette), traduction qui reste très aléatoire, puisque plusieurs indices laissent à penser que le journal sert souvent de support à certaines pratiques «solitaires ».

\section{Conclusion : Bagatelle, un révélateur}

Bagatelle, premier magazine féminin à proposer sur le marché un projet éditorial entièrement centré sur la question de la sexualité, peut apparaître comme un précurseur lorsqu'on constate l'importance prise aujourd'hui par 
les sujets sexuels comme arguments de vente dans la presse féminine. Toutefois, cette nouveauté n'a pas assuré sa survie. A force de prudence, Bagatelle risquait en effet, paradoxalement, la contradiction là où il cherchait à innover, de même qu'il proposait une relation incertaine à ses lectrices en définissant peu les modèles féminins qu'il envisageait, tout en personnalisant fortement la ligne éditoriale autour de sa rédactrice en chef. Cependant, l'expérience de Bagatelle montre que les tensions qui traversent son projet sont les tensions mêmes de la presse féminine, qu'il s'agisse des enjeux du positionnement ou des référents socioculturels mobilisés, et il est intéressant de noter que c'est précisément la recherche d'un discours différent sur la sexualité des femmes qui les fait apparaître. On peut penser que Bagatelle, en postulant l'existence d'un regard féminin sur la sexualité susceptible d'être partagé, c'est-à-dire d'une représentation stabilisée, reposait en fait la question de l'«identité féminine» qui justifierait l'existence d'une presse féminine, question qui reste ouverte à l'intérieur même de la presse qui se qualifie comme telle.

La spécialisation du contenu a peut-être accentué l'indétermination déjà à l'œuvre au sein de la presse féminine, dans la mesure où Bagatelle n'a sans doute pas envisagé précisément les lectrices auxquelles il entendait adresser son discours, et ce, malgré l'utilisation affichée des outils marketing. Ces outils s'avèrent être davantage un élément constitutif des stratégies de légitimation de l'existence du magazine, et ne s'inscrivent pas dans une démarche de structuration et de régulation d'un double marché. Ainsi, le sondage du numéro un, analysé plus haut, suggère un profil de lectrices potentielles favorables au fait de montrer des hommes nus, qui sont plutôt jeunes et dotées en capital économique et/ou culturel, tandis que Bagatelle cherche au contraire à s'adresser à des femmes de tous les âges et n'hésite pas à mêler les références culturelles, passant des stars du show business à la littérature galante des Lumières. On ne note pas, non plus, une réorientation progressive du magazine qui tienne compte des multiples enquêtes qu'il propose à son lectorat d'un numéro à l'autre. Ce positionnement en suspens, essentiellement fondé sur une offre originale, n'a sans doute pas contribué à convaincre les annonceurs (en dehors du secteur du Minitel rose et des agences de rencontre) qu'un titre centré sur la sexualité pouvait créer un univers de discours propre à favoriser la promotion de leurs produits. Mais il est possible, également, que les responsables du magazine n'aient pas voulu adopter une gestion marketing des contraintes du double marché, contraintes formulées sous la forme d' «attentes » du lectorat et des annonceurs, qui soumet tout choix éditorial aux études et au ciblage des lecteurs. 
Selon cette conception, des acteurs économiques interviennent sur ce marché afin d'organiser la rencontre entre une offre et une demande en vue de réaliser des bénéfices financiers. Cette conception ne convient pas forcément à la façon dont les journalistes appréhendent leur rôle vis-à-vis d'un lectorat et, dans le cas de Bagatelle, le souci des responsables de Bagatelle serait alors d'éviter qu'un cadrage trop important de la production rédactionnelle par les outils marketing freine l'innovation journalistique, car la démarche marketing élabore une conception en réalité purement commerciale du public. De même, la personnalisation aussi marquée du projet éditorial autour d'une figure journalistique unique, celle de la rédactrice en chef, pourrait alors révéler plus généralement le rôle déterminant et l'implication de l'équipe journalistique autour de ce projet.

Doit-on en déduire qu'il est impossible de proposer à un lectorat féminin un magazine exclusivement consacré à ce thème ? La façon dont les magazines féminins actuels traitent de la sexualité peut aussi être significative du tournant que Bagatelle n'a sans doute pas su prendre, en termes de segmentation du lectorat. En effet, au lieu de s'appuyer sur un lectorat généraliste mais concerné par une approche «osée » de la sexualité, ces titres qualifiés de seconde génération se caractérisent par une spécialisation du registre énonciatif et non des contenus, puisque la question sexuelle, même lorsqu'elle est partie prenante de ce registre, n'est qu'un sujet parmi d'autres. C'est à partir de ces positionnements discursifs qu'une segmentation du lectorat est alors réalisée. Mais, au rebours, on constate un appauvrissement de l'univers de la sexualité féminine, réduit à quelques figures très identifiées, quand il ne disparaît pas tout à fait des magazines féminins. On peut ainsi distinguer quatre tendances aujourd'hui à l'œuvre, qui s'adressent à des publics différents par leur inscription socioéconomique et/ou de leur appartenance à une classe d'âge. Ces tendances s'appuient sur différents types de discours : le discours de la "garce», le discours du «bien être» psychologique et corporel, le discours de la «femme à responsabilités » et le «féminin de société ». Jeune et Jolie, 20 ans, Jalouse appartiennent à des degrés divers à la première tendance et touchent un lectorat jeune. L'univers construit par les articles rappelle les publicités Kookaï, dans lesquelles des jeunes filles jouent avec des hommes miniatures, les soumettant à leurs désirs puérils (c'est la figure du «Girl Power »). Non seulement on est loin de l'érotisme adulte de Bagatelle, mais on retrouve une forme de "guerre des sexes » aux antipodes de son projet. Les magazines tels que Vital, Psychologies construisent, eux, l'image d'une lectrice en quête d'équilibre, personnel et conjugal, au sein duquel la 
sexualité occupe une place essentielle donnant lieu à de nombreux conseils pratiques et prescriptions «éthiques» (à travers des dossiers sur «les droits sexuels» par exemple, rappelant parfois certaines «résolutions» de Bagatelle comme «J'ai le droit de dire non ») mais ils quittent peu un registre sage et les scénarios sexuels déjà connus. Des titres comme Femme Active s'adressent, eux, aux cadres et aux chefs d'entreprise. Leurs contenus s'organisent autour d'un discours de la « réussite», de l'univers domestique à la sphère professionnelle. Enfin, le «féminin de société » $D S$ entend se distinguer des autres magazines féminins en traitant de sujets que l'on retrouve généralement dans les newsmagazines (Le Nouvel Observateur, L'Express, etc.), principaux concurrents sur le marché publicitaire, tout en conservant un «ton féminin ». Dans ces deux derniers types de presse, la sexualité disparait presque entièrement de l'univers féminin, comme si ce retrait constituait précisément une marque de «sérieux » au moment où les magazines généralistes (Elle, Cosmopolitan, Marie Claire...) multiplient les couvertures et les sujets pseudo-érotiques. De ce point de vue, Bagatelle a défriché, malgré ses contradictions, un territoire possible pour la presse féminine qui reste encore largement inexploré. 
BOZON A., GIAMI A. (1999), «Les scripts sexuels ou la mise en forme du désir. Présentation de l'article de J. Gagnon », Actes de la recherche en sciences sociales, 128 , juin, p. 69-72.

CHALVON-DEMERSAY S., PASQUIER D. (1990), Drôles de stars, Aubier.

DARDIGNA A.-M., (1974), Femmes - Femmes sur papier glacé, Coll. Cahiers libres, Maspéro.

DAMIAN B. (1995), Les dames du temps présent. Places et fonctions des études marketing dans la conception des discours de la presse féminine généraliste, Thèse réalisée sous la direction de B. Miège, Grenoble III.

DAMIAN B. (1997), La presse magazine féminine, in B. Lamizet et A. Silhem (sous la direction de), Dictionnaire encyclopédique des Sciences de l'information et de la communication, Ellipses/Editions marketing SA.

GAGNON J.H. (1999), «Les usages explicites et implicites de la perspective des scripts dans les recherches sur la sexualité », Actes de la recherche en sciences sociales, 128, juin, p. 73-79.

JOST F. (1992), Un monde à notre image, Méridiens/Klincksieck.

MAIGRET E. (1995), «'Strange grandit avec moi'. Sentimentalité et masculinité chez les lecteurs de bandes dessinées de super-héros », Réseaux, $\mathrm{n}^{\circ} 70$.

RUNTE-HELBING A. (1986), « Récits de vie dans les magazines féminins et féministes des années 1970 en Allemagne de l'Ouest », Cahiers de sémiotique textuelle, 8-9, 1986.

SOULEZ G. (1998), La médiation rhétorique à la télévision. Propositions pour l'analyse du présentateur comme orateur médiatique, Thèse réalisée sous la direction de N. Nel, Metz.

SOULEZ G. (1999), «La Tribune et l'Ecran », in Télévision, questions de forme, Colloque d'Aix-en-Provence, L'Harmattan, à paraître.

SOULEZ G. (2001), «La Rhétorique comme lien entre les théories. L'exemple de la crédibilité des journalistes à la télévision et à la radio ", XII ${ }^{e}$ Congrès des Sciences de l'Information et de la Communication, UNESCO, janvier, Actes à paraître.

SULLEROT E. (1966), La presse féminine. Les faits. La presse. L'opinion, Armand Colin, (seconde édition).

VERON E. (1984), «Quand lire, c'est faire : l'énonciation dans le discours de la presse écrite », Sémiotique II, IREP, p. 35-56.

VERON E. (1994) «De l'image sémiologique aux discursivités. Le temps d'une photo », Hermès, 13-14,, p. 45-64. 